\title{
Development of an Arithmetic Function using Euler's Phi Function and Study of its Properties
}

\author{
Nischal Maharjan, Bhupendra Budha \\ Department of Physics, St. Xavier's College, Tribhuvan University, Kathmandu, Nepal \\ Corresponding authors: nischal.maharjan@sxc.edu.np, bhupendra.budha@sxc.edu.np
}

Received: Jan 5, 2019

Revised: March 3, 2019

Accepted: March 7, 2019

\begin{abstract}
The central results of this article are $\mathrm{f}(\mathrm{n})=\frac{\varphi(\mathrm{n} !)}{\varphi\{(\mathrm{n}-1) !\}}$ equals to $(n-1)$ whenever ' $n$ ' is a prime number and it equals to (n) otherwise. These results can be used to determine and define the set of prime numbers. This result can also be used to realize the density of prime numbers in a given interval.
\end{abstract}

Keywords: Euler's phi function, prime numbers, arithmetic function.

\section{Introduction}

Euler Phi function: Euler's phi function, also known as Euler's totient function counts the number of positive integers which are less or equal to 'n' and are relatively prime to 'n'. It is denoted by $\varphi(n)$ or $\phi(n)[4,5]$.

Table 1: values of $\varphi(n)$ for the values of $\mathrm{n}$ from 1 to 10

\begin{tabular}{|l|l|l|l|l|l|l|l|l|l|l|}
\hline $\mathrm{n}:$ & 1 & 2 & 3 & 4 & 5 & 6 & 7 & 8 & 9 & 10 \\
\hline$\varphi(n):$ & 1 & 1 & 2 & 2 & 4 & 2 & 6 & 4 & 6 & 4 \\
\hline
\end{tabular}

Lemma 1 [3]: $\varphi(\boldsymbol{n})=\boldsymbol{n} \times \prod_{p \mid \boldsymbol{n}}\left(\mathbf{1}-\frac{\mathbf{1}}{\boldsymbol{p}}\right)$, where product is over distinct prime numbers 'p' divides ' $\mathrm{n}$ '.

Lemma 2 [2]: $\varphi\left(\boldsymbol{p}^{\boldsymbol{\alpha}}\right)=\boldsymbol{p}^{\boldsymbol{\alpha}}-\boldsymbol{p}^{\boldsymbol{\alpha}-\mathbf{1}}$ where 'p' is prime number and $\boldsymbol{\alpha} \in \boldsymbol{Z}^{+}, \varphi(\boldsymbol{p})=\boldsymbol{p}-\mathbf{1}$, for $\alpha=1$

Lemma 3: $\varphi(\boldsymbol{m} \cdot \boldsymbol{n})=\varphi(\boldsymbol{m}) . \varphi(\boldsymbol{n})$, where ' $\mathrm{m}$ ' and 'n' are relatively prime numbers $[1,2]$.

Lemma 4: $\varphi(\boldsymbol{m} \cdot \boldsymbol{n})=\varphi(\boldsymbol{m}) \cdot \varphi(\boldsymbol{n}) \cdot \frac{\boldsymbol{d}}{\varphi(\boldsymbol{d})}$, where $\mathrm{d}=\operatorname{gcd}(\mathrm{m}, \mathrm{n})[2]$.

\section{Proposition and Proof}

2.1 Proposition: Let us define an arithmetic function $f(n)$ as $f(n)=\frac{\varphi(n !)}{\varphi\{(n-1) !\}}$. Then we propose, 


$$
f(\boldsymbol{n})=\left\{\begin{array}{cc}
\boldsymbol{n}-\mathbf{1}, & \text { if } \boldsymbol{n} \text { is prime } \\
\boldsymbol{n}, & \text { otherwise }
\end{array}\right.
$$

\subsection{Proof:}

\subsubsection{Proof for $1^{\text {st }}$ case:}

Here, ' $\mathbf{n}$ ' is a prime number. Let there exist ' $\mathbf{r}$ ' distinct primes between $\mathbf{1}$ and (n-1)! such that $(n-1) !=p_{1}^{k_{1}} \cdot p_{2}^{k_{2}} \ldots p_{r}^{k_{r}}$, where $p_{1}, p_{2}, \ldots, p_{r}$ are ' $\mathrm{r}$ ' distinct primes (using unique factorization theorem). We know, $n>p_{1}, p_{2}, \ldots, p_{r}$.

Hence, $\mathrm{n}$ and $(\mathrm{n}-1)$ ! are relatively prime. Then we have,

$$
f(n)=\frac{\varphi(n !)}{\varphi\{(n-1) !\}}=\frac{\varphi\{n \cdot(n-1) !\}}{\varphi\{(n-1) !\}}=\frac{\varphi(n) \cdot \varphi\{(n-1) !\}}{\varphi\{(n-1) !\}}=\varphi(n)=n-1 \text {, using lemmas } 2 \text { and } 3 .
$$

Therefore, the $1^{\text {st }}$ case is true.

\subsubsection{Proof for $2^{\text {nd }}$ case:}

Subcase I: When ' $n$ ' is a composite number.

Here, $\mathrm{n}$ is a composite number. Let $\mathrm{n}$ be composed of ' $\mathrm{s}$ ' distinct primes such that $n=p_{1}^{k_{1}} \cdot p_{2}^{k_{2}} \ldots p_{s}^{k_{s}}=\prod_{i=1}^{s} p_{i}^{k_{i}}$, where $p_{1}, p_{2}, \ldots, p_{s}$ are 's' distinct primes.

We know (n-1)! is composed of every number from 1 to (n-1). This implies that (n-1)! is composed of every prime number between 1 to $(n-1)$. Let ' $d$ ' be $\operatorname{gcd}\{n,(n-1) !\}$. Then we have, $d=p_{1}^{k_{1}^{\prime}} \cdot p_{2}^{k_{2}^{\prime}} \ldots p_{s}^{k_{s}^{\prime}}=\prod_{i=1}^{s} p_{i}^{k_{i}^{\prime}}$, where $k_{i}^{\prime} \leq k_{i}$ as $d \leq n$.

It implies

$$
\begin{gathered}
f(n)=\frac{\varphi(n !)}{\varphi\{(n-1) !\}}=\frac{\varphi\{(n) \cdot(n-1) !\}}{\varphi\{(n-1) !\}}=\frac{\varphi(n) \cdot \varphi\{(n-1) !\}}{\varphi\{(n-1) !\}} \cdot \frac{d}{\varphi(d)}=\frac{\varphi(n) \cdot d}{\varphi(d)}=\frac{n \cdot \prod_{p \mid n}\left(1-\frac{1}{p}\right) \cdot d}{d \cdot \prod_{p \mid d}\left(1-\frac{1}{p}\right)} \\
=\frac{n \cdot\left(1-\frac{1}{p_{1}}\right) \cdot\left(1-\frac{1}{p_{2}}\right) \cdot\left(1-\frac{1}{p_{3}}\right) \ldots \cdot\left(1-\frac{1}{p_{r}}\right)}{\left(1-\frac{1}{p_{1}}\right) \cdot\left(1-\frac{1}{p_{2}}\right) \cdot\left(1-\frac{1}{p_{3}}\right) \ldots \cdot\left(1-\frac{1}{p_{r}}\right)}=n
\end{gathered}
$$

(Using lemmas 1 and 4)

Subcase II: When $\mathrm{n}=1$ (i.e., neither prime nor composite)

$$
f(1)=\frac{\varphi(1 !)}{\varphi(1-1) !}=\frac{\varphi(1 !)}{\varphi(0 !)}=1
$$

Therefore, from subcase I and II, it is clear that $2^{\text {nd }}$ case is true. 


\section{Applications}

\subsection{Determination of Prime Numbers}

We know, $f(\boldsymbol{n})=\left\{\begin{array}{cc}\boldsymbol{n}-\mathbf{1}, & \text { if } \boldsymbol{n} \text { is prime } \\ \boldsymbol{n}, & \text { otherwise }\end{array}\right.$

From these results, we can generate the set of prime numbers.

Let $\eta$ be the set of numbers generated by $f(n)=\frac{\varphi(n !)}{\varphi\{(n-1) !\}} \cdot f(n)=n-1$ when ' $\mathrm{n}$ ' is prime number. As we know, 2 and 3 are the only two consecutive prime numbers. So, for $n=3$ in $f(n)$, we get 2 which is the only prime number in set $\eta$.

Let $Z^{+}$be the set of natural numbers. Then we can define a new set $P$ as

$P=Z^{+}-\eta=$ set of all prime numbers $-\{2\}=$ set of all odd prime numbers

Then, we can define set of all prime numbers as union of $P$ and $\{2\}$.

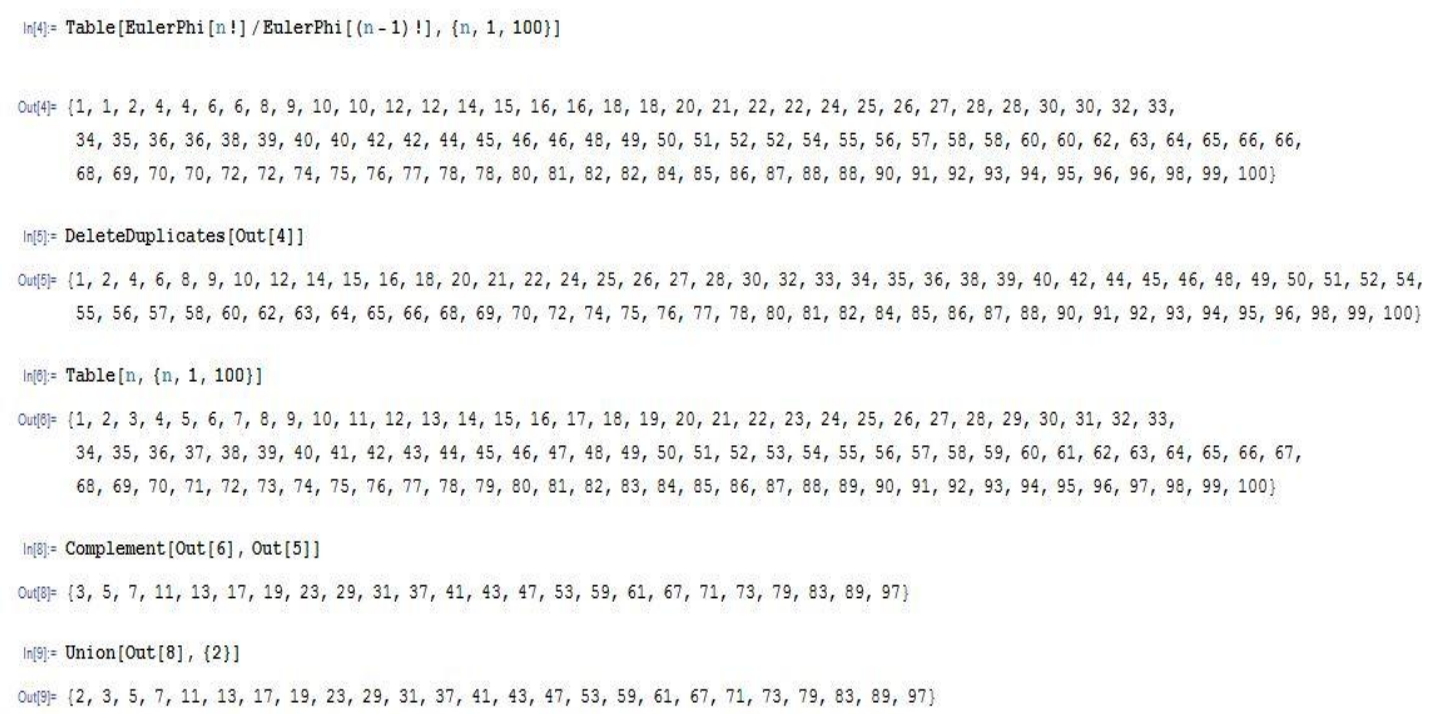

Fig. 1: Set of prime numbers from 1 to 100 formed using $f(n)$ with the help of Mathematica

\subsection{Realization of Density of Prime Numbers in a Given Interval}

Let $T$ be the table of numbers generated by $f(n)=\frac{\varphi(n !)}{\varphi\{(n-1) !\}}$. For all composite numbers ' $n$ ' in $f(n)$, we get ' $\mathrm{n}$ ' i.e. if we plot $n v s f(n)$ graph using only composite numbers and pass line through all the points, then we will get a straight line passing through origin. But for prime numbers ' $n$ ' in $f(n)$, we get ' $n-1$ ' and if we plot all $n v s f(n)$ graph using all the natural numbers then due to presence of prime numbers, there will be no straight line passing through 
origin as numbers generated by prime numbers in $f(n)=\frac{\varphi(n !)}{\varphi\{(n-1) !\}}$ will break the straight line and form a zigzag line.

So we can derive a conclusion that if there are many zigzags in the graph of certain interval of natural numbers, then the interval is densely packed with prime numbers, if there are not many zigzags in the graph of certain interval of natural numbers, then the interval is not densely packed with prime numbers and if the line is straight in the graph of certain interval of natural numbers, then the interval does not contain any prime numbers. Hence, by observing the graph, we can realize the density of prime numbers in given interval. Few examples of $n$ vs $f(n)$ graph plotted using the help of Mathematica are shown below:

\section{a. Interval which is densely packed with Prime Numbers}

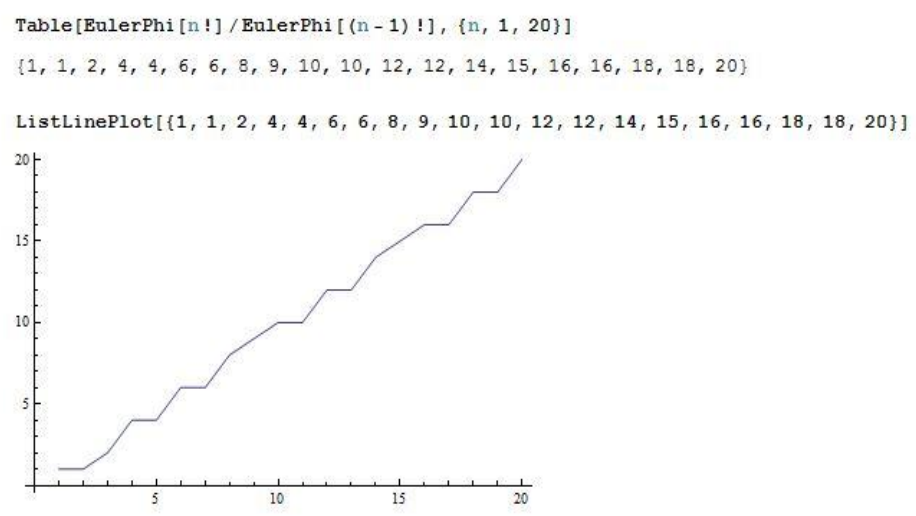

Fig. 2: Line plot of the function $f(n)$ ranging from 1 to 20

\section{b. Interval which is not densely packed with Prime Numbers}

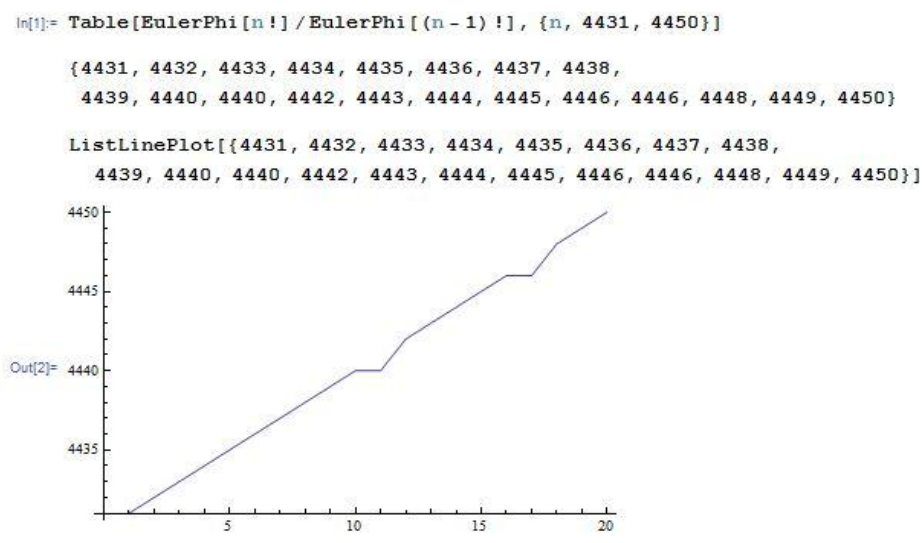

Fig. 3: Line plot of the function $f(n)$ ranging from 4431 to 4450 


\section{c. Interval with no Prime Numbers}

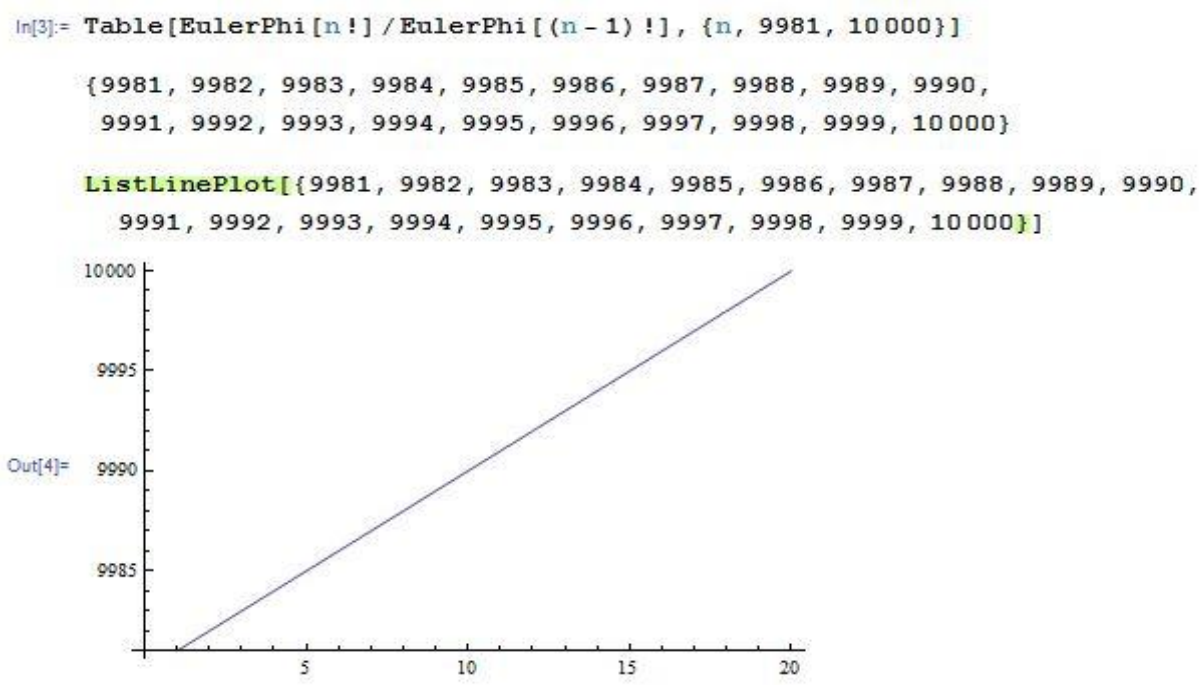

Fig. 4: Line plot of the function $f(n)$ ranging from 9981 to 10000

\section{Conclusion}

The main result of this article is $f(\boldsymbol{n})=\left\{\begin{array}{cc}\boldsymbol{n}-\mathbf{1}, & \text { if } \boldsymbol{n} \text { is prime } \\ \boldsymbol{n}, & \text { otherwise }\end{array}\right.$ Hence, this result can be exploited to construct an algorithm to generate the set of prime numbers and to observe the density of prime numbers in any given interval of natural numbers.

Acknowledgement: We would like show our gratitude to Dr. Ajaya Singh, Central Department of Mathematics, TU for assisting us during the completion of our work. Also we would like to express deep gratitude to Prof. Dr. Gyan Thapa, Institute of Engineering, TU, Nepal for valuable guidance and encouragement.

\section{References}

[1] Apostol TM (1976), Introduction to Analytic Number Theory, Springer-Verlag, New York, Inc, USA.

[2] Burton DM (2006), Elementary number theory, Tata McGraw-Hill Education.

[3] Chorge S and Vargas J (2013), Proof of Euler's $\varphi$ (Phi) Function Formula, Rose-Hulman Undergraduate Mathematics Journal, 14(2): 91-95.

[4] Long CT (1972), Elementary Introduction to Number Theory, Lexington: D. C. Heath and Company, LCCN, 77171950.

[5] Pettofrezzo AJ and Byrkit DR (1970), Elements of Number Theory, Prentice Hall International, London, Inc, England. 